\title{
Childhood infectious diseases and old age cognitive functioning: a nationally representative sample of community-dwelling older adults
}

\author{
A. Rotstein (1) and S. Z. Levine \\ Department of Community Mental Health, Faculty of Social Welfare and Health Sciences, University of Haifa, Haifa, Israel
}

ABSTRACT

Background: Cumulative evidence suggests that health-related risk factors during midlife and old-age are associated with cognitive impairment. However, studies are needed to clarify the association between early-life risk factors and impaired cognitive functioning to increment existing knowledge.

Objective: To examine the association between childhood infectious diseases and late-life cognitive functioning in a nationally representative sample of older adults.

Participants: Eligible respondents were 2994 community-dwelling individuals aged 65-85.

Measurements: Cognitive functioning was assessed using the Mini-Mental State Examination (MMSE). Childhood infectious diseases (i.e. chicken pox, measles, and mumps) were self-reported. The study covariates were age, sex, highest educational level achieved, smoking status, body mass index, and depression. The primary statistical analysis examined the association between the number of childhood infectious diseases and total MMSE scores, accounting for all study covariates. Regression models of progressive complexity were examined for parsimony. The robustness of the primary results was tested in 17 sensitivity analyses.

Results: The most parsimonious model was a linear adjusted model (Bayesian Information Criterion $=$ 12646.09). Late-life cognitive functioning significantly improved as the number of childhood infectious diseases increased $(\beta=0.18 ; 95 \% \mathrm{CI}=0.11,0.26 ; p<0.001)$. This effect was not significantly attenuated in all sensitivity analyses.

Conclusion: The current study results are consistent with prior ecological findings indicating that some childhood infectious diseases are associated with better cognitive functioning in old-age. This points to an early-life modifiable risk factor associated with older-life cognitive functioning. Our results may reflect selective mortality and/or beneficial effects via hormetic processes.

Key words: cognition, childhood illness, epidemiology

\section{Introduction}

Impaired cognitive functioning among communitydwelling older adults is estimated to have a prevalence of $18.3 \%$ in the UK (Rait et al., 2005), ranging between $8.7 \%$ and $22.2 \%$ in the United States (Plassman et al., 2008; Langa et al., 2008). Impaired cognitive functioning is associated with increased risks of Alzheimer's disease (Bäckman et al., 2005) and mortality (Langa et al., 2008). The identification

Correspondence should be addressed to: Anat Rotstein, Department of Community Mental Health, Faculty of Social Welfare and Health Sciences, University of Haifa, Haifa 3498838, Israel. Phone: + 972522629707 ; Fax + 97237617374. Email: rotstein.anat@gmail.com Received 22 Mar 2020; revision requested 12 May 2020; revised version received 31 May 2020; accepted 20 Jun 2020. First published online 24 July 2020. of risk factors and preventative strategies are primary objectives in research on impaired cognitive functioning in older adults (Brayne, 2007; Treichler and Jeste, 2019). Indeed, general health-related risk factors have been identified during midlife and old age (Launer, 2005; Launer, 2007; Kaufman and Perales-Puchalt, 2019; Lee et al., 2019; Shaaban et al., 2019; Tu et al., 2020; Walker et al., 2019). Nonetheless, a recent shift has seen studies that examine the role of early-life risk factors to increment current knowledge of risk factors (Grainger et al., 2019; Launer, 2007; Whalley et al., 2006; Williamson and Leroi, 2019).

Few observational studies have examined the association between health in early-life and late-life cognition. Two studies found that retrospective reports of better global childhood health were 
associated with improved cognitive functioning in old age (Luo and Waite, 2005; Yi et al., 2007). Three others studies reported a null association (Barnes et al., 2012; Yount, 2016; Zhang et al., 2018). A single study examined the association between childhood infectious diseases and late-life cognitive functioning and showed mixed disease effects (Case and Paxson, 2009). This study used an ecological design to examine childhood health based on region-level historical data and linked it with performance on cognitive tests during adulthood. Ecological studies are valuable for assessing the global disease burden, yet may not accurately reflect the true association for a given group (Lilienfeld, 1983; Piantadosi et al., 1988). In addition, ecological studies raise concerns of the ecological fallacy (Robinson, 1950), whereby lower and higher levels of abstraction are not interchangeable (i.e. implying individual-level characteristics from collective ones may be erroneous).

Competing views exist concerning the long-term impact of childhood infectious diseases. Exposure to childhood infectious diseases has been shown to be associated with both negative (Case and Paxson, 2009; Dalman et al., 2008) and positive health outcomes in later life (Alexander et al., 2000; Amirian et al., 2016; Case and Paxson, 2009). Negative outcomes may be explained via direct effects of pathogens and indirect effects of inflammatory response (Khandaker et al., 2012). Positive outcomes may be attributed to mild stress-induced repeated stimulation of protective mechanisms in cells and organisms which has a wide range of health-promoting and life span-extending effects (Rattan, 2008), and/or selective mortality.

The current study aims to examine the direction of the association between common childhood infectious diseases and late-life cognitive functioning in a nationally representative sample of communitydwelling older adults.

\section{Methods}

\section{Design and data source}

The current study cohort was based on a nationally representative sample of community-dwelling individuals aged 65 and over from the Republic of Ireland. Data were obtained from the Irish Longitudinal Study on Ageing (Kenny, 2018). This largescale, nationally representative, aging study was conducted in Ireland. The current data were derived from interviews, undertaken between January 2016 and December 2016. The response rate was $62.0 \%$. The study was approved by the Faculty of Health Sciences Research Ethics Committee in Trinity College Dublin. Further details of sampling and study design have been described elsewhere (Whelan and Savva, 2013).

\section{Participants}

Eligible respondents for this study included community-dwelling individuals aged 65-85 $(N=3434)$. People with known or suspected dementia were not recruited. A subsample of people who did not have any information on past illnesses of any sort were removed $(N=440)$. Therefore, the final sample was 2994 people.

\section{Cognitive functioning}

Cognitive functioning was assessed with the MiniMental State Examination (MMSE) (Folstein et al., 1975). The MMSE is one of the most widely used research and screening measures of cognitive functioning in aging populations (Folstein et al., 1975, 1983). It is easy to administer, available in over 15 languages (Steis and Schrauf, 2009), and has high levels of acceptability by health professionals and researchers as a diagnostic instrument (Nieuwenhuis-Mark, 2010). The scale has 20 items to assess orientation, recall, attention, calculation, language, and visuospatial abilities. It is divided into two sections, the first of which requires vocal responses only and covers orientation, memory, and attention; the maximum score is 21 . The second part tests cognitive abilities to name, follow verbal and written commands, write a sentence spontaneously, and copy a polygon; the maximum score is nine. The maximum overall score is 30 , with higher scores representing better cognitive functioning (Folstein et al., 1983). Questions were asked and scored immediately. The test was not timed. The tester was instructed first to make the individual comfortable, to establish rapport, to praise successes, and to avoid pressing on items which the individual finds difficult.

In the current study, MMSE scores were computed, based on the original instructions (Folstein et al., 1975) for each of the five subscales: orientation, registration, attention and calculation, recall, and language. Total scores were computed as well. For the primary analysis, the total test scores were not dichotomized into categories of impaired cognitive functioning and intact cognitive functioning because dichotomizing leads to information lost, increased risk of a false positive and may seriously underestimate the extent of variation in outcome such that individuals close to but on opposite sides of the cut point are characterized very differently rather than similarly (Altman and Royston, 2006). However, for robustness, when recomputing the sensitivity analysis, the total test scores were 
dichotomized into categories of impaired and intact cognitive functioning.

\section{Childhood infectious diseases}

The childhood infectious diseases selected for this study were chickenpox, measles, and mumps. These were chosen because they are viral diseases, infection with which are followed by an enduring immunity (Simpson, 1952) and were common at the time of our sample's childhood years due to recurrent outbreaks (London and Yorke, 1973). Childhood infectious diseases were assessed self-reported by asking participant if they have had chicken pox, measles, and mumps in childhood. Responses were classified as "yes" (score of 1), "no" (score of 0), or "don't know" (coded as missing values). A total number of childhood infectious diseases score was computed for each person as the sum of the reported diseases of chicken pox, measles, and mumps. The total score ranged from 0 (having had no diseases) to 3 (having had all three diseases).

\section{Covariates}

The following covariates were included in the analyses to adjust for potential confounding, model effect modification and to define subgroups of particular interest with possible differential effects on cognitive functioning. The covariates considered (and entered in the following order) were demographics, metabolic, and psychiatric. Demographic covariates were age at the time of data collection and sex (van der Flier and Scheltens, 2005), highest education achieved [primary or none, secondary, third, or higher (Solfrizzi et al., 2004; Tervo et al., 2004; Tyas et al., 2007)], and smoking status at the time of data collection [classified as smoker or nonsmoker (Swan and Lessov-Schlaggar, 2007)]. The metabolic covariate was body mass index categories [0-25, 25-30, 30-40, 40+(Atti et al., 2008)]. The psychiatric variable was depression (Butters et al., 2008) as measured by the Composite International Diagnostic Interview (Robins et al., 1988). This established instrument is widely used for assessing a clinical diagnosis of major depression in epidemiological and clinical studies. Respondents received binary scores depending on whether they had fulfilled criteria for a major depressive episode in the last 12 months or not.

\section{Analytic approach}

First, missing values were examined, and the data were imputed accordingly using MICE (Multivariate Imputation by Chained Equations; van Buuren and Groothuis-Oudshoorn, 2010) in R package for multivariate imputation by chained equations. Second, sample characteristics were computed.

Third, the primary statistical analysis tested the association between the total MMSE scores as a function of the number of childhood infectious diseases using regression models. The assumptions of the regression models were tested. Visual inspection of residual figures was performed in order to reveal deviations from homoscedasticity or normality. An inspection for normality of error terms followed using a histogram and probability plots of the residuals. Independence of the error term was examined through a scatter plot of residuals by the predicted values to show that no discernible association existed. Next, multiple regression analysis models were computed. Regression models were computed in ascending complexity and tested without adjustment and adjusted for confounding of age at the time of data collection, sex, highest education achieved, smoking status, body mass index, and depression. Models were numbered as follows. The first model accounted for a linear effect of the number of childhood infectious diseases on MMSE scores (model 1 hereafter). The second model accounted for a linear effect of the number of childhood infectious diseases, age, sex, education level, smoking status, body mass index, and depression on MMSE scores (model 2 hereafter). The third model accounted for a quadratic effect of the number of childhood infectious diseases on MMSE scores (model 3 hereafter). The fourth model accounted for a quadratic effect of the number of childhood infectious diseases, age, sex, education level, smoking status, body mass index, and depression on MMSE scores (model 4 hereafter). The fifth model accounted for a cubic effect of the number of childhood infectious diseases on MMSE scores (model 5 hereafter). The sixth model accounted for a cubic effect of the number of childhood infectious diseases, age, sex, education level, smoking status, body mass index, and depression on MMSE scores (model 6 hereafter).

Fourth, the six models were compared for parsimony based on the Bayesian Information Criterion (BIC) for model selection (Schwarz, 1978), similar to prior research (Rotstein et al., 2018). Lower BIC values represent more parsimonious models and so are a better fit to the data. The best fitting model was then chosen based on the lowest BIC score and plotted using the ggplot2 library (Wickham, 2011). All analyses were computed in $R$ ( $R$ Core Team, 2018).

Fifth, the robustness of the primary results was tested in 17 sensitivity analyses. The most parsimonious regression model was recomputed in subgroups 
Table 1. Sample characteristics

\begin{tabular}{|c|c|}
\hline & $\mathrm{M}(\mathrm{SD}) / N(\%)$ \\
\hline $\begin{array}{l}\text { Total Mini-Mental State Examination } \\
\text { score }(0-30)\end{array}$ & $28.37(2.14)$ \\
\hline History of chicken pox (score of 1 ) & $2029(67.8 \%)$ \\
\hline History of measles (score of 1 ) & $2684(89.7 \%)$ \\
\hline History of mumps (score of 1 ) & $1866(62.3 \%)$ \\
\hline $\begin{array}{l}\text { Total number of childhood infectious } \\
\text { diseases }(0-3)\end{array}$ & $2.20(0.95)$ \\
\hline Age $(65-85$ years $)$ & $73.50(6.18)$ \\
\hline Sex (male) & $1372(45.8 \%)$ \\
\hline Education & $\begin{array}{l}\text { Primary/none: } 906 \\
\text { (30.3\%) } \\
\text { Secondary: } 1119 \\
\text { (37.4\%) } \\
\text { Third/higher: } 969 \\
\quad(32.4 \%)\end{array}$ \\
\hline Smoking status (smoker) & $265(8.85 \%)$ \\
\hline Body mass index & $\begin{array}{c}0-24.99: 1001 \\
(33.4 \%) \\
25-29.99: 1334 \\
(44.6 \%) \\
30-39.99: 628 \\
(21.0 \%) \\
40+: 31(1.0 \%)\end{array}$ \\
\hline $\begin{array}{l}\text { Depression (a major depressive } \\
\text { episode in the last } 12 \text { months) }\end{array}$ & $99(3.3 \%)$ \\
\hline
\end{tabular}

with differential effects on cognitive functioning. First, the most parsimonious model was recomputed without sex as a covariate for females then males, since females are at greater risk for impaired cognitive functioning (van der Flier and Scheltens, 2005). Second, the most parsimonious model was recomputed for persons aged $65-75$ then aged $75-85$, since cognitive functioning is related to age (van der Flier and Scheltens, 2005). Third, the most parsimonious model was computed for each of the three diseases (i.e. chicken pox, measles, and mumps) separately to show their individual effect on cognitive functioning. Fourth, the most parsimonious model was computed using a saturated model in which dummy variables for one, two, or three childhood infectious diseases were entered as covariates. Fifth, the most parsimonious model was computed for each of the MMSE subscales (i.e. orientation, registration, attention and calculation, recall, and language) separately. Sixth, the most parsimonious model was computed without education to account for effects of mediation. Seventh, possible methodological confounders were considered. Although dichotomizing may be problematic (Altman and Royston, 2006), for robustness and because dichotomous models have increased clinical lure, the most parsimonious model was recomputed dichotomized to account for intact cognitive functioning versus impaired cognitive functioning. Impaired cognitive functioning was defined using a cutoff score of 1.5 standard deviations below the mean score of the MMSE (Palmer et al., 2002). Additionally, the most parsimonious model was recomputed for observed data with missing values and compared to the primary analysis based on imputed data (Sterne et al., 2009).

\section{Results}

\section{Data imputation and sample characteristics}

The data were imputed using multiple imputations because simulation studies showed the technique to be robust even if the data are not missing at random (Sinharay et al., 2001). The analytic sample consisted of 2994 older adults. The sample had a mean age of $73.50(\mathrm{SD}=6.18)$ and $54.2 \%(N=1,622)$ were female. The average MMSE score was 28.37 $(\mathrm{SD}=2.14)$. Most of the participants had a history of measles $(89.7 \%$; $N=2,684)$, chicken pox $(67.8 \%$; $N=2,029)$, and/or mumps $(62.3 \% ; N=1,866)$. See Table 1 for all sample characteristics.

\section{Primary statistical analysis: cognitive functioning and the number of childhood infectious diseases}

Comparison of the six regression models showed that model 2 was the most parsimonious $(\mathrm{BIC}=$ 12646.09; see supplementary Table S1 for comparison of BIC values for all regression models published as supplementary material online attached to the electronic version of this paper at https://doi.org/ $10.1017 / \mathrm{S} 1041610220001404)$. This model accounted for the linear effect of the number of childhood infectious diseases on MMSE scores (Figure 1), adjusted for age, sex, education level, smoking status, body mass index, and depression. Late-life cognitive functioning improved as the number of childhood infectious diseases increased. Each disease incremented the MMSE score by 0.18. For two diseases, the MMSE total score increased by 0.36 . Having had three diseases increased the MMSE score by 0.54 . See Table 2 for model statistics.

\section{Sensitivity analyses}

Model 2, accounting for the linear effect of the number of childhood infectious diseases, age, education level, smoking status, body mass index, and depression on MMSE scores, was recomputed for all sensitivity analyses. A significant effect of childhood infectious diseases was found for males $(N=1372)$, females $(N=1622)$, persons aged $65-75$ $(N=1828)$, persons aged $75-85(N=1166)$, each of 
Table 2. Model 2 statistics

\begin{tabular}{lrrr}
\hline Variable & Coefficient & $95 \% \mathrm{Cl}$ & P-value \\
Age & -0.09 & $-0.10 ;-0.07$ & $<0.001$ \\
Sex (female) & 0.05 & $0.09 ;-0.20$ & 0.47 \\
Education (secondary) & 0.79 & $0.61 ; 0.96$ & $<0.001$ \\
Education (third/higher) & 1.20 & $1.02 ; 1.38$ & $<0.001$ \\
Smoking (smoker) & -0.53 & $-0.78 ;-0.28$ & $<0.001$ \\
BMl (25-29.99) & 0.10 & $-0.07 ; 0.26$ & 0.24 \\
BMl (30-39.99) & -0.02 & $-0.22 ; 0.18$ & 0.85 \\
BMl (40+) & -0.35 & $-1.06 ; 0.36$ & 0.33 \\
Depression & -0.32 & $-0.72 ; 0.08$ \\
No. diseases & 0.18 & $0.11 ; 0.26$ & 0.11 \\
\hline
\end{tabular}

Note. Model 2 is the linear effect of the number of childhood infectious diseases, age, sex, education level, smoking status, body mass index, and depression on Mini-Mental State Examination scores.

Sex reference group $=$ male.

Education reference group $=$ primary/none.

Smoking status reference group $=$ non-smoker.

Body mass index reference group $=0-24.99$.

Depression reference group $=$ not having had a major depressive episode in the last 12 months.

Abbreviations: $\mathrm{BMI}=$ Body Mass Index; No. diseases $=$ the number of childhood infectious diseases.

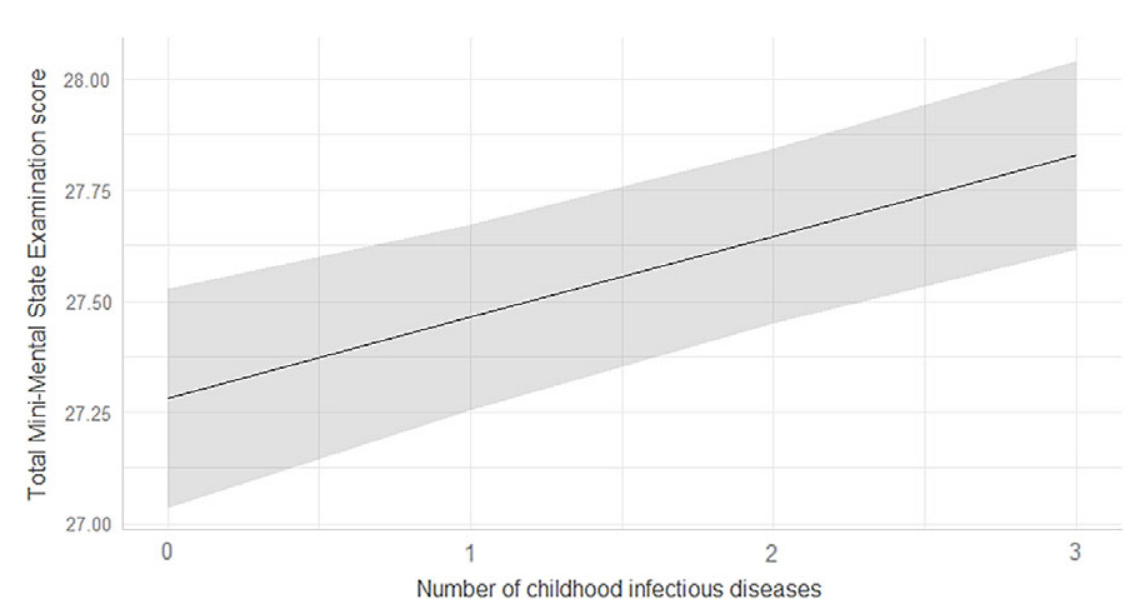

Figure 1. The linear adjusted effect of the number of childhood infectious diseases on Mini-Mental State Examination scores.

Note. For this adjusted model, having had one disease increases the Mini-Mental State Examination score by 0.18 , all other covariates equal. Having had two diseases increases the Mini-Mental State Examination total score by 0.36 , all other covariates equal. Having had three diseases increases the score by 0.54 , all other covariates equal.

the childhood infectious diseases separately (chicken pox, measles, and mumps), a saturated model (in which the effect size increased as the number of childhood infectious diseases increased), each subscale of the MMSE separately, a dichotomized regression model (intact cognitive functioning vs. impaired cognitive functioning), a model without education, and observed data with missing values (in which 191 people were excluded due to missingness; $N=2803$ ). See supplementary Tables S2-S17 for model statistics (published as supplementary material online attached to the electronic version of this paper). 


\section{Discussion}

The current study examined the association between childhood infectious diseases and old age cognitive functioning, among community-dwelling older adults, using data from a representative national sample. The primary results show that late-life cognitive functioning improved as the number of childhood infectious diseases increased. Specifically, for each additional disease, there was an improvement in cognition reflecting a 0.18 MMSE total point increase. The primary result was not statistically significantly attenuated in a series of sensitivity analyses, including four subgroups with potentially differential cognitive functioning, two methodological confounders, two alternative models, each infectious disease and each MMSE subscale examined. The strongest effects were found among females and among those aged $75-85$ years.

The current study results are consistent with prior ecological findings showing that some childhood infectious diseases (i.e. influenza) are associated with better cognitive functioning (i.e. successful counting) in old age (Case and Paxson, 2009). The literature provides related examples of early-life infectious diseases having a protective effect on health in later life. For instance, the varicella zoster virus that causes chickenpox is consistently reported to have an inverse association with glioma (Amirian et al., 2016). Similarly, measles and/or combined childhood infections (chicken pox, measles, mumps, pertussis, and rubella) were found protective for Hodgkin's disease (Alexander et al., 2000).

Consistent with prior studies that have reported positive long-term health effects of childhood infectious diseases, our results contribute to identifying modifiable risk factors positively related to older-life cognitive functioning. Tentative explanations of our results are (I) selective mortality; and/or (II) that low levels of exposure to harmful agents may have beneficial effects via hormetic processes (Rattan, 2008). Future studies may identify possible mechanisms.

The current study has several limitations. First, selective mortality may have occurred, biasing the current study results. Second, our results are restricted to chickenpox, measles, and mumps. Future research is warranted to ascertain the effects of other childhood infectious diseases on cognitive functioning in old age. Third, childhood infectious diseases were assessed through self-reports which are less reliable than testing for serum antibodies (Mortimer, 1978). Specifically, higher rates of prevalence for actual mumps antibodies are expected since one person in three who contracts mumps does not present any symptoms (Mortimer, 1978). Still, prior evidence has suggested that these assessments of childhood health have reasonably good reliability and validity (Haas, 2007). In addition, past epidemiological studies have found similar prevalence rates as those reported in the current study for chickenpox, measles, and self-reported mumps (Mortimer, 1978; Pollock and Golding, 1993; Stocks, 1928). Nonetheless, it should be noted that a selection bias may have occurred as respondents with better cognitive function may have recalled their childhood experience of infectious diseases more accurately. Fourth, the MMSE relies on an interviewer administration and rating which may introduce further biases. Although the interviewer is given specific instructions for administration, differences resulting from the skill and style of the interviewer in eliciting answers and in scoring the answers given by the subject exist (Bowie et al., 1999). Fifth, the sample did not include individuals with verified diagnoses of dementia or mild cognitive decline. Future studies may focus on individuals with and without dementia to extend the current findings.

\section{Conclusions}

In summary, despite its limitations, the current study draws on a large representative sample and is the first to examine the individual and cumulative effects of common childhood infectious diseases (chickenpox, measles, and mumps) on late-life cognitive functioning. The study results show a consistent positive association between the number of childhood infectious diseases and late-life cognitive functioning. Childhood infectious diseases may be a future direction for modifiable risk factors related to older-life cognitive functioning.

\section{Conflicts of interest}

None.

\section{Description of authors' role}

Both authors designed the study, conducted the statistical analyses, interpreted the data, and prepared the manuscript.

\section{Acknowledgments}

Based on data collected through the Irish Longitudinal study on Ageing. Accessed via the Irish Social Science Data Archive -www.ucd.ie/issda.

\section{Supplementary material}

To view supplementary material for this article, please visit https://doi.org/10.1017/S1041610220001404 


\section{References}

Alexander, F. E. et al. (2000). Risk factors for Hodgkin's disease by Epstein-Barr virus (EBV) status: prior infection by EBV and other agents. British fournal of Cancer, 82, $1117-1121$.

Altman, D. G. and Royston, P. (2006). The cost of dichotomising continuous variables. BMF, 332(7549), 1080.

Amirian, E. S. et al. (2016). History of chickenpox in glioma risk: a report from the glioma international case-control study (GICC). Cancer Medicine, 5, 1352-1358.

Atti, A. R. et al. (2008). Late-life body mass index and dementia incidence: nine-year follow-up data from the Kungsholmen Project. Fournal of the American Geriatrics Society, 56(1), 111-116.

Bäckman, L. et al. (2005). Cognitive impairment in preclinical Alzheimer's disease: a meta-analysis. Neuropsychology, 19(4), 520.

Barnes, L. L. et al. (2012). Effects of early-life adversity on cognitive decline in older African Americans and whites. Neurology, 79(24), 2321-2327.

Bowie, P. et al. (1999). Should the Mini Mental State Examination be used to monitor dementia treatments? The Lancet, 354(9189), 1527-1528.

Brayne, C. (2007). The elephant in the room-healthy brains in later life, epidemiology and public health. Nature Reviews Neuroscience, 8(3), 233-239.

Butters, M. A. et al. (2008). Pathways linking late-life depression to persistent cognitive impairment and dementia. Dialogues in Clinical Neuroscience, 10(3), 345.

Case, A. and Paxson, C. (2009). Early life health and cognitive function in old age. American Economic Review, 99(2), 104-109.

Dalman, C. et al. (2008). Infections in the CNS during childhood and the risk of subsequent psychotic illness: a cohort study of more than one million Swedish subjects. American Fournal of Psychiatry, 165, 59-65.

Folstein, M. F. et al. (1975). "Mini-mental state": a practical method for grading the cognitive state of patients for the clinician. Fournal of Psychiatric Research, 12(3), 189-198.

Folstein, M. F. et al. (1983). The mini-mental state examination. Archives of General Psychiatry, 40(7), 812.

Grainger, S. A. et al. (2019). An investigation into early-life stress and cognitive function in older age. International Psychogeriatrics, 1-5. doi: 10.1017/S1041610219001583

Haas, S. A. (2007). The long-term effects of poor childhood health: An assessment and application of retrospective reports. Demography, 44(1), 113-135.

Kaufman, C. S. and Perales-Puchalt, J. (2019). Cardiovascular contributions to dementia: beyond individual risk factors. International Psychogeriatrics, 31(10), 1387-1389.

Kenny, R. A. (2018). The Irish Longitudinal Study on Ageing (TILDA), 2012-2013.

Khandaker, G. M. et al. (2012). Childhood infection and adult schizophrenia: a meta-analysis of population-based studies. Schizophrenia Research, 139(1-3), 161-168.

Langa, K. M. et al. (2008). Trends in the prevalence and mortality of cognitive impairment in the United States: is there evidence of a compression of cognitive morbidity? Alzheimer's and Dementia, 4(2), 134-144.
Launer, L. J. (2005). The epidemiologic study of dementia: a life-long quest? Neurobiology of Aging, 26(3), 335-340.

Launer, L. J. (2007). Next steps in Alzheimer's disease research: interaction between epidemiology and basic science. Current Alzheimer Research, 4(2), 141-143.

Lee, E. E. et al. (2019). High prevalence and adverse health effects of loneliness in community-dwelling adults across the lifespan: role of wisdom as a protective factor. International Psychogeriatrics, 31(10), 1447-1462.

Lilienfeld, A. M. (1983). Practical limitations of epidemiologic methods. Environmental Health Perspectives, $52,3-8$

London, W. P. and Yorke, J. A. (1973). Recurrent outbreaks of measles, chickenpox and mumps: I. Seasonal variation in contact rates. American fournal of Epidemiology, 98(6), 453-468.

Luo, Y. and Waite, L. J. (2005). The impact of childhood and adult SES on physical, mental, and cognitive well-being in later life. The fournals of Gerontology Series B: Psychological Sciences and Social Sciences, 60(2), S93-S101.

Mortimer, P. P. (1978). Mumps prophylaxis in the light of a new test for antibody. British Medical fournal, 2(6151), 1523-1524.

Nieuwenhuis-Mark, R. E. (2010). The death knoll for the MMSE: has it outlived its purpose? Fournal of Geriatric Psychiatry and Neurology, 23(3), 151-157.

Palmer, K. et al. (2002). Differential evolution of cognitive impairment in nondemented older persons: results from the Kungsholmen Project. American fournal of Psychiatry, 159(3), 436-442.

Piantadosi, S. et al. (1988). The ecological fallacy. American fournal of Epidemiology, 127(5), 893-904.

Plassman, B. L. et al. (2008). Prevalence of cognitive impairment without dementia in the United States. Annals of Internal Medicine, 148(6), 427-434.

Pollock, J. I. and Golding, J. (1993). Social epidemiology of chickenpox in two British national cohorts. Fournal of Epidemiology and Community Health, 47(4), 274-281.

Rait, G. et al. (2005). Prevalence of cognitive impairment: results from the MRC trial of assessment and management of older people in the community. Age and Ageing, 34(3), 242-248.

Rattan, S. I. S. (2008). Hormesis in aging. Ageing Research Reviews, 7(1), 63-78.

Robins, L. N. et al. (1988). The Composite International Diagnostic Interview: an epidemiologic instrument suitable for use in conjunction with different diagnostic systems and in different cultures. Archives of General Psychiatry, 45(12), 1069-1077.

Robinson, W. (1950). Ecological correlations and the behavior of individuals, American Sociological Review, 15, 351-357.

Rotstein, A. et al. (2018). Age of onset and quality of life among males and females with schizophrenia: a national study. European Psychiatry, 53, 100-106.

Schwarz, G. (1978). Estimating the dimension of a model. The Annals of Statistics, 6(2), 461-464.

Shaaban, C. E. et al. (2019). Independent and joint effects of vascular and cardiometabolic risk factor pairs for risk of all-cause dementia: a prospective population-based study. International Psychogeriatrics, 31(10), 1421-1432. 
Simpson, R. H. (1952). Infectiousness of communicable diseases in the household (measles, chickenpox, and mumps). Lancet, 549-554.

Sinharay, S. et al. (2001). The use of multiple imputation for the analysis of missing data. Psychological Methods, 6(4), 317.

Solfrizzi, V. et al. (2004). Vascular risk factors, incidence of MCI, and rates of progression to dementia. Neurology, 63(10), 1882-1891.

Steis, M. R. and Schrauf, R. W. (2009). A review of translations and adaptations of the Mini-Mental State Examination in languages other than English and Spanish. Research in Gerontological Nursing, 2(3), 214-224.

Sterne, J. A. C. et al. (2009). Multiple imputation for missing data in epidemiological and clinical research: potential and pitfalls. BMF, 338, b2393.

Stocks, P. (1928). A study of the epidemiology of measles. Annals of Eugenics, 3(3-4), 361-398.

Swan, G. E. and Lessov-Schlaggar, C. N. (2007). The effects of tobacco smoke and nicotine on cognition and the brain. Neuropsychology Review, 17(3), 259-273.

R Core Team (2018). R: A Language and Environment for Statistical Computing, $R$ Foundation for Statistical Computing. Austria: R Core Team.

Tervo, S. et al. (2004). Incidence and risk factors for mild cognitive impairment: a population-based three-year follow-up study of cognitively healthy elderly subjects. Dementia and Geriatric Cognitive Disorders, 17(3), 196-203.

Treichler, E. B. and Jeste, D. V. (2019). Cognitive decline in older adults: applying multiple perspectives to develop novel prevention strategies. International Psychogeriatrics, 31(7), 913-916.

Tu, L. et al. (2020). Trajectories of cognitive function and their determinants in older people: 12 years of follow-up in the Chinese Longitudinal Healthy Longevity Survey. International Psychogeriatrics, 32(6), 765-775.
Tyas, S. L. et al. (2007). Transitions to mild cognitive impairments, dementia, and death: findings from the Nun Study. American fournal of Epidemiology, 165(11), 1231-1238.

van Buuren, S. and Groothuis-Oudshoorn, C. G. (2010). Mice: multivariate imputation by chained equations in $\mathrm{R}$. Fournal of Statistical Software, 1-68.

van der Flier, W. M. and Scheltens, P. (2005). Epidemiology and risk factors of dementia. Fournal of Neurology, Neurosurgery and Psychiatry, 76(Suppl. 5), v2-v7.

Walker, K. A. et al. (2019). Association of midlife to late-life blood pressure patterns with incident dementia. $\mathcal{F} A M A$, 322(6), 535-545.

Whalley, L. J. et al. (2006). A life-course approach to the aetiology of late-onset dementias. The Lancet Neurology, 5(1), 87-96.

Whelan, B. J. and Savva, G. M. (2013). Design and methodology of the Irish Longitudinal Study on Ageing. Fournal of the American Geriatrics Society, 61, S265-S268.

Wickham, H. (2011). ggplot2. Wiley Interdisciplinary Reviews: Computational Statistics, 3(2), 180-185.

Williamson, W. and Leroi, I. (2019). Thinking about dementia: is childhood too early? International Psychogeriatrics, 31(12), 1689-1690.

Yi, Z. et al. (2007). The association of childhood socioeconomic conditions with healthy longevity at the oldest-old ages in China. Demography, 44(3), 497-518.

Yount, K. M. (2016). Gender, resources across the life course, and cognitive functioning in Egypt. In: N. Chamlou and M. Karshenas (Eds.), Women, Work and Welfare in the Middle East and North Africa (pp. 107-133). Singapore: World Scientific.

Zhang, Z. et al. (2018). The long arm of childhood in China: early-life conditions and cognitive function among middleaged and older adults. Fournal of Aging and Health, 30(8), 1319-1344. 